\title{
Progreso Tecnológico y Desarrollo Económico: Una Aproximación Marxista para el Enfoque Humano del Desarrollo
}

Roberto Argelio Frías Jiménez ${ }^{*}$

Alan Lemos

Cualquier acercamiento al problema que nos ocupa requerirá de un adecuado enfoque metodológico si es que pretende, en alguna medida, ser legítimo. Tal enfoque debe permitir la realización del ejercicio intelectual que la naturaleza del problema objeto de análisis exige. De acuerdo con esto es necesario también aclarar el significado que tienen aquellos conceptos con los que se pretende operar en la realización del análisis y que son asumidos como claves en el proceso de nuestra exposición.

Los problemas de la relación entre desarrollo tecnológico y desarrollo humano, los vemos a través del prisma de la relación entre los problemas sociales de la ciencia y la tecnołogía y, para los efectos prácticos de nuestro trabajo, serán enfocados teniendo en cuenta dos niveles: Primero aquel que está intrínsecamente asociado al desarrollo de la cicncia o de la tecnología y que tiene un matiz gnoseológico, es decir un problema o conjunto de ellos que se enmarcan dentro de la clásica dicotomía leniniana acerca de que no hay diferencias de principios entre el fenómeno y la esencia, sino entre lo conocido y lo que falta por conocer, brecha que jamás será ganada, pero que como exigencia social es siempre el principal estímulo al desarrollo tanto de la ciencia como de la tecnología. Segundo, el que se refiere a lo que se ha dado en llamar impactos de la ciencia y la tecnología en la estructura social. ${ }^{1}$ Este nivel será nuestro principal objeto de exposición.

Esos impactos se exponen aquí en relación con los procesos de desarrollo de la actividad científica y tecnológica cuyos componentes son el conjunto de elementos denominados invención, innovación y cambio. En este trabajo se le dedica mayor atención a los procesos de innovación tecnológica y no porque no se considere el problema social de la ciencia, sino porque el hecho real de que ésta se haya convertido en una fuerza

\footnotetext{
'Dr., Professor do Departamento de Economia da Universidad de Matanzas, Cuba.

"M.C., Professor do Departamento de Economia da Universidade Federal de Roraima, Brasil.
}

I Asumimos aqui el concepto estructura social en el sentido que Marx le da en el Prólogo a la Contribución a la Crítica de la Economía Política. El uso de este término se ha prestado últimamente a determinadas confusiones, sobre todo en personas habituadas al clásico esquema que identifica el concepto estructura con el de relaciones de producción o económicas. Nosotros lo hemos tomado del trabajo "El desafío de los aflos 90: Diez Nuevas Tendencias". Edición de Adolfo Castillo, María Cruz Alonso y José A. Díaz, pp.1314. 
productiva directa, es decir, en un instrumento de transformación productiva de las fuerzas de la naturaleza y de la propia sociedad, reducen tanto el momento de la ciencia y de la tecnología que es casi imposible distinguirlos como procesos independientes. Por otro lado, como señala Nuñez: "La tecnología contemporánea es un producto de la ciencia", y alude a cierto empalme de la tecnología con las disciplinas científicas que las han engendrado. ${ }^{2}$ Basándonos en estas afirmaciones que compartimos totalmente hemos tomado la opción válida de centrarnos en la tecnología para, a través de ella, presuponer a la ciencia.

En lo que al enfoque metodológico se refiere queremos significar que el que se aplique no puede verse como una externalidad que le viene al objeto de análisis desde afuera, sino que aunque parezca absurdo (y decimos parezca porque en realidad no lo es), el enfoque debe nacer de la naturaleza del propio objeto de análisis. En el caso que nos ocupa, el objeto sobre el que versan nuestras reflexiones, son las relaciones sociales estudiadas desde la perspectiva de los impactos que sobre ellas ejercen el desarrollo de la ciencia y de la tecnología.

De acuerdo con ésto, dentro de la amplia gama de enfoques que la ciencia conoce para analizar, estudiar e investigar este nexo, consideramos que el que mejor se aviene a las características de nuestro campo de estudio es, sin lugar a dudas, el enfoque marxista. ${ }^{3}$ Aunque el objetivo de este trabajo no es argumentar la afirmación que hemos hecho, si vale la pena decir que el desarrollo de la ciencia y la tecnología y sus impactos sobre las relaciones sociales, constituyen una regularidad a la que como tal le es aplicable el principio científico de la repetición.

Sobre la base de este razonamiento hemos formulado la siguiente hipótesis de trabajo: "Si existe una relación estrecha entre los llamados problemas sociales de la ciencia y la tecnología, como objeto de nuestro análisis, y el enfoque marxista para el estudio de las relaciones sociales como método de investigación, cumpliéndose así uno de los principios fundamentales de la concepción de Marx acerca de la unidad entre el objeto y el método de análisis, entonces es posible descubrir y argumentar desde posiciones científicas los impactos que sobre las relaciones sociales tienen el desarrollo de la ciencia y la tecnología."

Trataremos ahora de develar, según nuestra interpretación, ese punto nodal que representa la unidad entre el objeto y el método de la investigación.

El problema social, como objeto de la investigación está planteado en Marx en función de la realización de lo que él define como esencia humana. Esta problemática es fundamental para poder comprender el verdadero significado del ya aceptado concepto de

\footnotetext{
Jorge Núñez Jover. Ciencia - Tecnología - Desarrollo Social. Problemas Sociales de la Ciencia y la tecnología. GESOCTY, pp.88.

${ }^{3}$ Cuando se habla del Enfoque marxista nos referimos al sistema de principios que se derivan de la concepción de Marx elaborada por él en su estudio de la sociedad capitalista y formulada suscintamente en el Prólogo de la Contribución a la Crítica de la Economía Política y ampliamente desplegada en su obra cumbre El Capital.
} 
humumsmo marxista. El mismo, como es ampliamente conocido, se contrapone al concepto smithiano de la naturaleza humana y de su homo economicus, pilares sobre los wales se crigió una concepción del hombre y del orden social correspondiente, donde mesumtamente se desarrollan sus cualidades identificatorias: egoísmo, espíritu nctcantilista, interés privado, propensión a consumir, a ahorrar, etc. Esta idea es limdancntal para, en nuestra opinión, poder distinguir a qué se refiere el concepto de lo lmumano cuando es utilizado para calificar el desarrollo.

Para Marx, la esencia humana "[...] no es algo abstracto inherente a cada individuo In, en su realidad, el conjunto de las relaciones sociales." 4

Para explicar desde posiciones científicas los problemas inherentes al desarrollo lustírico de esa esencia humana (del conjunto de las relaciones sociales en su realidad), Marx "L o hizo destacando de los diversos campos de la vida de la sociedad el de la conomia, destacando de todas las relaciones sociales, las relaciones de producción, por se las fundamentales, las primarias, las que determinan todas las demás."

Obsérvese que ahora nos estamos moviendo en el plano de la relación entre dos conceptos claves: El concepto de relaciones sociales, cuyo conjunto conforma la esencia lummana y el concepto de relaciones de producción o económicas, cuyo conjunto conforma la cscncia económica del sistema en cuestión y entre las que se establece un nexo causal donde las primeras se explican por las segundas.

11 proceso que explica claramente esta cuestión es desarrollado por Marx en el f'rolugo de la Contribución a la Crítica de la Economía Política, donde formula la hipritesis de que el desarrollo histórico de la esencia humana, no se puede explicar por si musmo, sino por el contrario, sus causas radican en las condiciones materiales de vida de los hombres, en las relaciones de producción como relaciones que los hombres contraen en la producción social de su vida y cuyo conjunto conforma la estructura económica de la sociedad (esencia económica). ${ }^{6}$

Es nạcesario destacar una observación realizada por Lenin, en la que está la clave para comprenter cómo se articula, en el análisis, el problema de la ciencia y de la iecnología. Se trata de que el propósito del análisis marxista es proporcionar un criterio objetivo y formular un criterio científico. Al primero se refiere el destacar a las relaciones de producción como estructura de la sociedad, al segundo "la posibilidad de aplicar a estas relaciones [...] la repetición." 7

\footnotetext{
' Carlos Marx. Tesis sobre Fewerbach. Tesis No. 6. En Carlos Marx y F. Engels, Obras Escogidas, en tres tomos, tomo 1. Lditorial Progreso, 1973, pp. 9
}

' VII cnin. Quiénes Son los Amigos del Publo y cómo Luchan Contra la Socialdemocracia. Editorial Progreso, Moscu, 1978, pp. 13.

" ('arlos Marx. Prólogo de la Contribución a la Crítica de la Economía Politica. En Obras Escogidas, en tres lomos, tomo 1. Editorial Progreso, Moscú, 1973, pp 517 - 518.

Idem nota 5, pp. 15 
He aquí porqué del sistema (conjunto) de las relaciones sociales Marx aísla una de sus partes para, a partir de ella explicar el todo. Esta parte son, precisamente, las relaciones de producción o económicas. El criterio científico general de la repetición permite descubrir lo que es constante, reiterado, causal, regular en la actividad humana y esto no es otra cosa que la producción y reproducción de la vida biológica y social del propio hombre y tanto una como la otra tienen como precedente la producción y reproducción de bienes materiales mediante el trabajo humano. Siendo éste un concepto clave, Marx le dedica un amplio análisis que abarca los trabajos desde la Ideología Alemana hasta El Capital. ${ }^{8}$ El trabajo como actividad específicamente humana, es analizado como la unidad de Medios y Fines. Desde la perspectiva de los primeros se destacan sus elementos constitutivos del trabajo como Proceso:

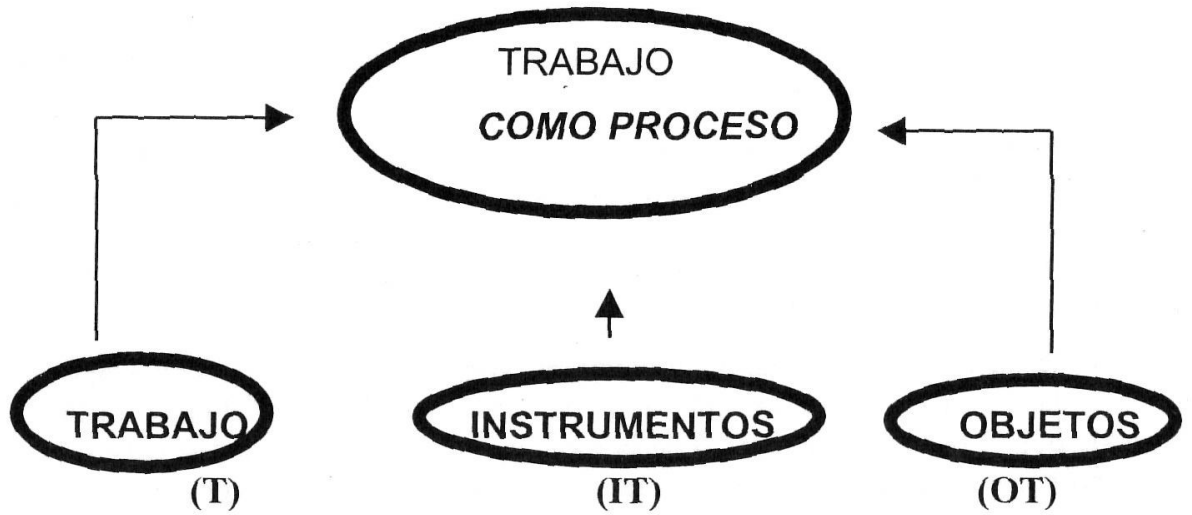

Entre estos tres elementos se da un tipo de relación que por sus características se distingue sustancialmente, tanto de las económicas o de producción como de las sociales. Son esas relaciones las que comúnmente se clasifican como tecnológicas y expresan el vínculo entre el hombre y el objeto de la transformación mediante los instrumentos.

Hay que tener en cuenta que ellas no agotan totalmente el contenido de la tecnología, pues aún hay que observar que existe, además, una relación entre hombres a través de la cual ocurre el proceso de transmisión de la experiencia productiva, técnica, organizativa, etc., entre unos y otros. Este aspecto es de suma importancia para poder comprender los procesos de innovación mediante la así llamada transferencia de tecnología. El siguiente gráfico resume la idea anterior:

\footnotetext{
${ }^{5}$ Ver C.Marx. Ideología Alemana. Editora Política, La Habana, 1979 y El Capital, tomo I, sección III, Cap. I, epigrafe 1 , pp 74 y 86.
} 


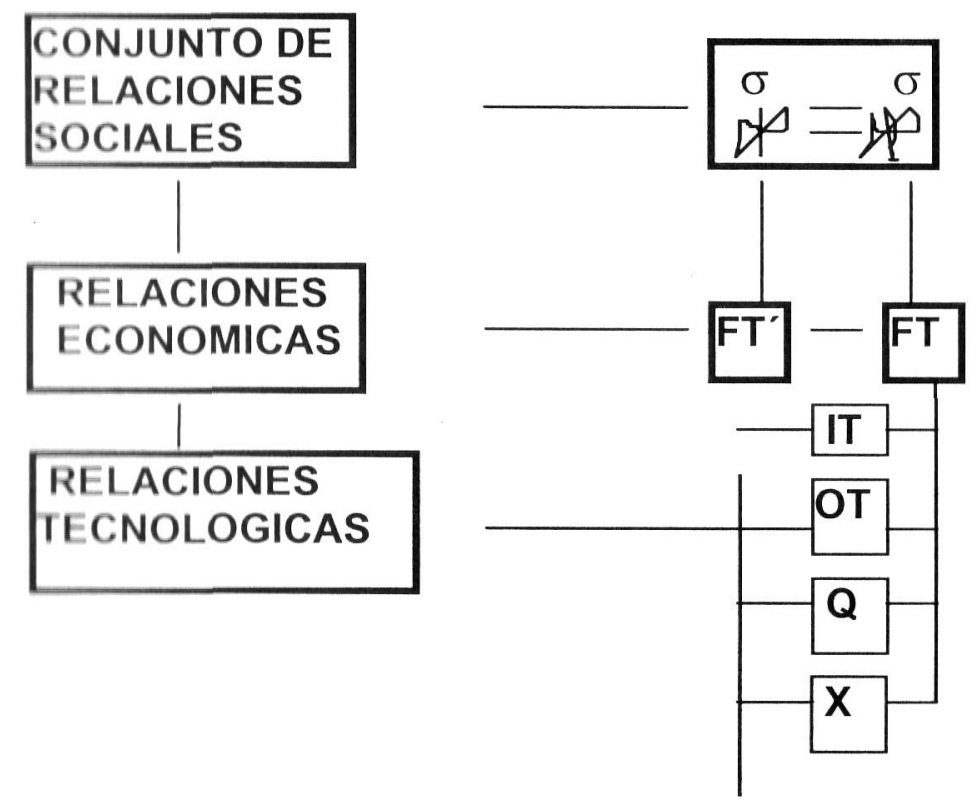

L « 4 relaciones de carácter tecnológico incluyen, además, otros momentos muy importantes; a los que se refieren:

- II saber hacer, enmarcado en la relación hombre - objeto.

- 11 saber hacer cada vez mejor, enmarcado también en la relación, pero ahora desde una Perspectiva dinámica (de desarrollo).

- La transferencia, enmarcada en la relación hombre - hombre y que incluye a su veh, dos momentos muy importantes:

a)Saber captar el saber hacer de terceros

b)Saber asimilar lo captado Lo dicho podríamos representarlo ampliando el gráfice anterior: 


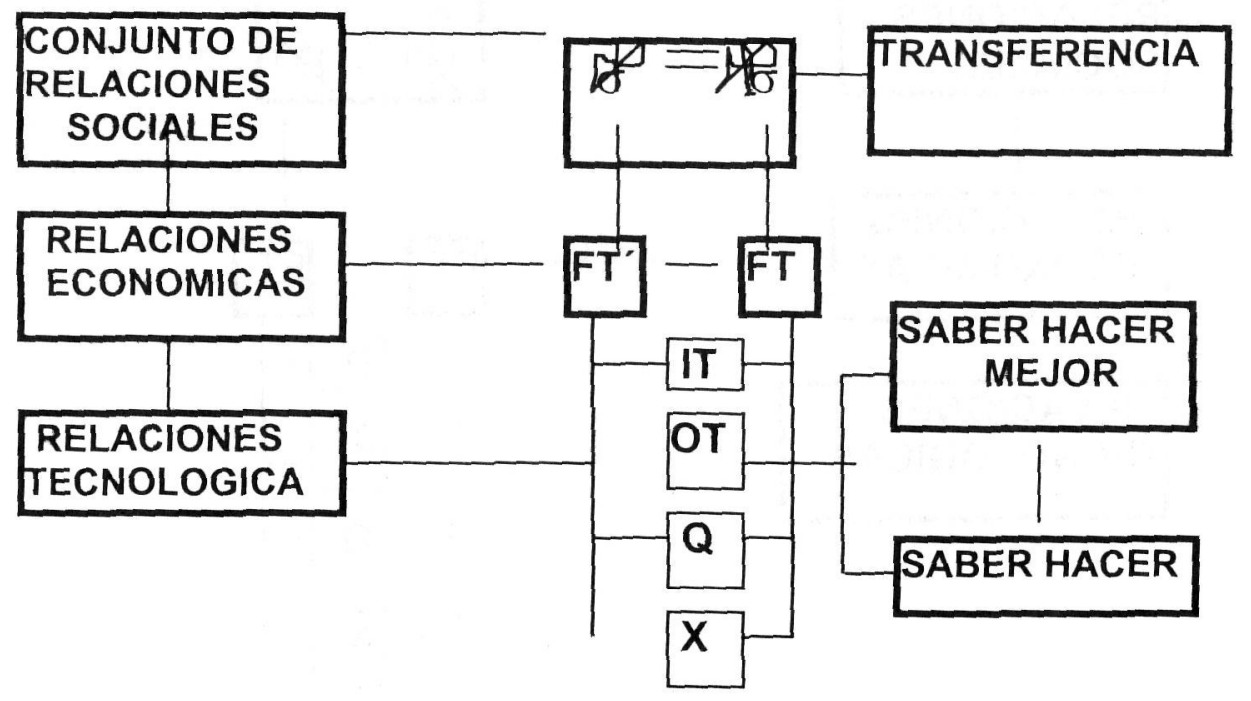

El gráfico muestra algunos conceptos claves de la teoría marxista que son necesarios considerar en el análisis.

Las relaciones entre hombres están al nivel de las relaciones sociales, pero cuando éstas se estudian en el nivel de las relaciones económicas, el concepto hombre aparece reducido a uno de sus lados, es decir, al concepto Fuerza de Trabajo. Esta, articulada con los Medios de Producción (IT + OT combinados), nos da el concepto marxista de Fuerzas Productivas, que como puede apreciarse en el esquema están asociadas, a su vez, al concepto de tecnología.

Nos parece que a lo largo del análisis se aprecia claramente la existencia de una estrecha relación entre lo que constituye nuestro objeto de estudio y el enfoque marxista de la investigación, idea que enunciamos en la hipótesis de trabajo que formulamos al inicio del trabajo. Es Lenin quien, a nuestro modo de ver, mejor destaca el nexo al que hacemos referencia: "[...] sólo reduciendo las relaciones sociales (esencia humana) a las de producción (esencia económica) y estas últimas al nivel de las fuerzas productivas 
racionalidad humana, económica y tecnológica. Lo dicho hasta aquí queda resumido en el gráfico siguiente:

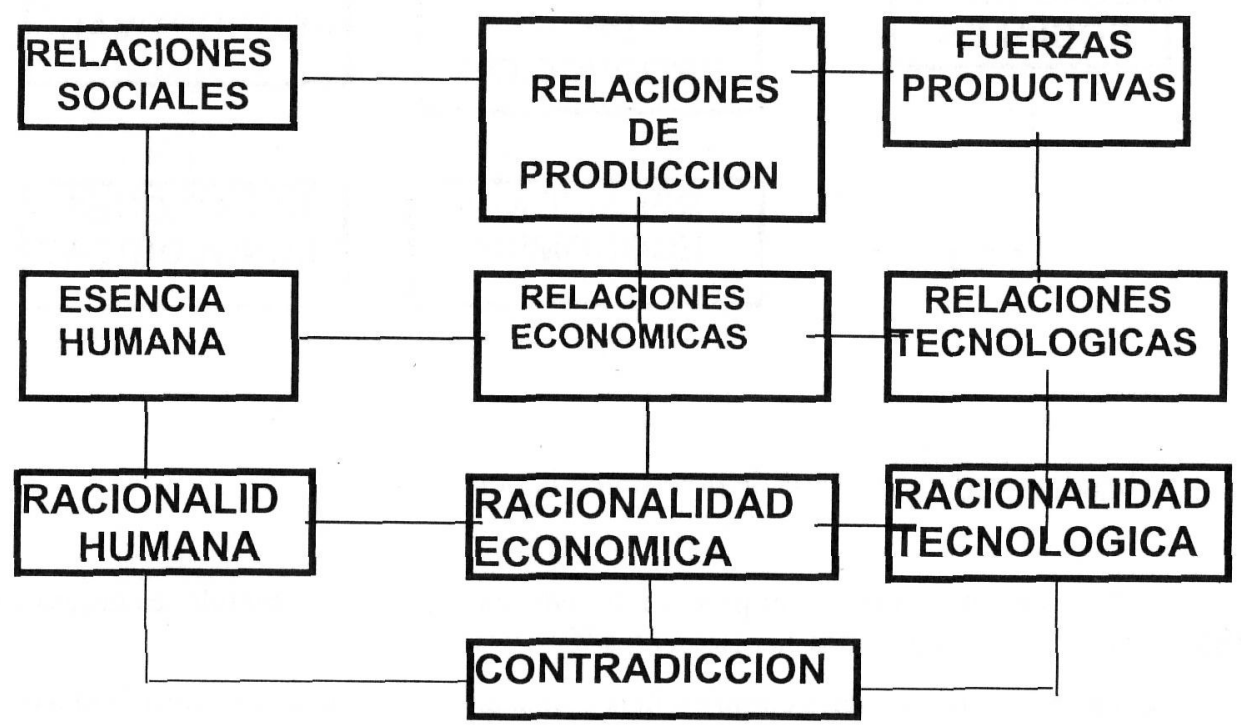

3. Las diferentes interpretaciones que se han hecho de esa interrelación presentan, en su mayoría, serias deficiencias. La exhaltación de la racionalidad humana (humanismo), en más de una ocasión ha hecho olvidar el análisis y las conclusiones de Marx desde la Ideología Alemana hasta Crítica al Programa de Gotha, donde aclara que el comunismo, como régimen (orden) social, y espacio ideal donde podrá realizarse en toda su plenitud la esencia humana, sólo puede ser el resultado de un alto desarrollo de las fuerzas productivas, es decir de las así llamadas relaciones tecnológicas.

Aún en vida de Engels, éste tuvo que asumir la defensa de la concepción marxista frente a los criterios bersteinianos sobre el determinismo económico. Constancia de ello es 
Ait cartia a thech de 1890, donde realiza un serio intento por el explivar el significado de "lo economico como determinante en última instancia". Esta corriente de pensamiento fiive aegmipano al socialismo mundial hasta su estrepitoso derrumbe y desmontaje.

I1 cucchat relación con el determinismo económico se ha desarrollado también cl detemintmo tecnológico, sobre cuya base se han estructurado las teorías de la medenizacion, cuyo remate es un capitalismo a ultranza, ahistórico y eterno y las teorias Acerca de la mposibilidad de que el socialismo se alcance en países con bajo nivel de denamollode las fucrzas productivas.

I 1 smgular de esta problemática es que casi todos los que sostienen una u otra pobicion las sustentan en mayor o menor medida tomando al vuelo una u otra cita de Mark

Nosotros pensamos que la mejor alternativa no consiste en ir pendularmente de un pinto a utro, ya hemos hablado del principio marxista de la unidad entre el objeto y el métedo 11 primero está constituido por la realidad social, frente a ella nos posicionamos enif ef enfoçuc marxista y descubrimos allí qué es válido y qué no lo es. En esto consiste, a miestru modo de ver, el carácter creador de la aplicación de tan monumental modelo fentico Ilacer to contrario es tratar de introducir en la realidad un estado de cosas

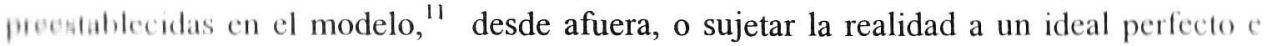
impreciso por no poder abarcar totalmente a la realidad y esto tiene más de hegelianismo puro que de marxismo creador aunque se haga muchas veces en su nombre.

\section{1) Infocque Marxista, Tecnología y los Modelos de Desarrollo}

Agui se incursiona en el Modelo marxista y se demuestran sus particularidades, asi como el papel que juega el progreso técnico en el movimiento de las estructuras economicas, que son precisamente el objeto de investigación en Marx. Establecemos un

In nuestra opinión tratar de ajustar la realidad al modelo, como casi siempre se intenta, es un imposible prâtico los modelos, cualquicra que ellos sean, cumplen funciones determinadas entre las que podemos menetemar la de servir de instrumentos para ayudar explicar la realidad que se pretende investigar, o do inducit ain modo determinado de pensamiento para enfrentar el objeto de investigación. En la práctica se puede apreciar que muchas veces se identifica a la realidad con el modelo ideal y esto provoca profundas rupturas cntre la primera y la segunda con consecuencias sociales nefastas. No debemos olvidar que la precisión de un modelo sc logria, precisamente, reflejando la realidad de modo impreciso puesto que el mismo no puede (al menos hasta ahora con todo el avance tecnológico que la humanidad ha registrado) agotar la realidad en toda su iratieamente magotable riqueza de fenómenos, matices, etc. 
puente entre el sistema categorial marxista y los nuevos conceptos con que se analizan hoy los problemas del desarrollo y el papel que en ello juega la Tecnología, así por ejemplo se introducen los conceptos de paradigma tecnológico y de tecnología disponible, para a partir de ello valorar la relación entre países avanzados y atrasados, desarrollados y subdesarrollados. En esta parte se expondrán algunas de las transformaciones que se dan en el ámbito de las estructuras sociales como consecuencia del proceso de innovación tecnológica.

Como quiera que lo dicho en el párrafo anterior se sitúa, de hecho, en el orden de algunos principios metodológicos, conviene que aclaremos a qué relaciones o estructuras sociales hacemos alusión. Nos referimos específicamente a relaciones de carácter cultural, económico, político, ideológico, filosófico, etc., tanto en el plano interno como en el marco de las relaciones entre países. Establecemos el nexo orgánico que existe entre estas relaciones y aquellas que tienen que ver con el desarrollo de la tecnología y sus cuatro pilares fundamentales:

\section{PILARES FUNDAMENTALES DEL DESARROLLO TECNOLOGICO}

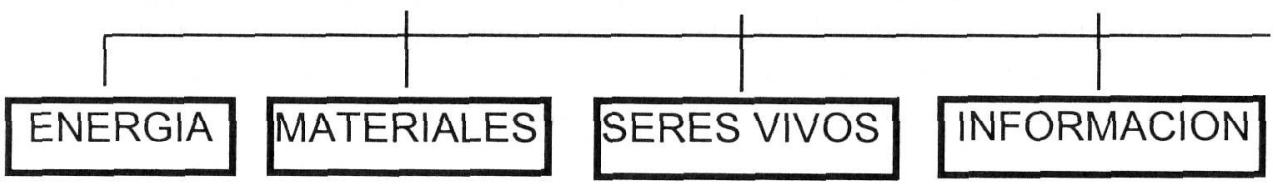

Para, a partir de esta interrelación explicar los cambios que se dan en las así llamadas estructuras sociales.

Por otra parte, es necesario destacar que la hipótesis marxista expuesta en la Contribución a la Crítica de la Economía Política es probada y demostrada en el análisis del modo de producción capitalista, cuyos resultados son expuestos por Marx en su obra cumbre El Capital. Señalamos esto por cuanto ello indica claramente el nexo esencial que existe entre desarrollo tecnológico y desarrollo histórico del capitalismo hasta nuestros días. [Marx (1962) y (1973)] 
In consecuencia con lo dicho, los problemas analizados aquí responden a la lógica econónica interna del capitalismo, a su mecanismo innato de funcionamiento y ha determinadas especificidades de su funcionamiento.

La ciencia económica conoce varios modelos o esquemas teóricos a partir de los cuales y con cuya ayuda se ha estudiado el nexo anteriormente enunciado. Toda la obra de Mark, El Capital, es un modelo de este tipo que está compuesto por las estructuras claves simuientes:

El Mecanismo de Acción de la Ley del Valor, el cual puede verse representado en el gráfico siguiente:

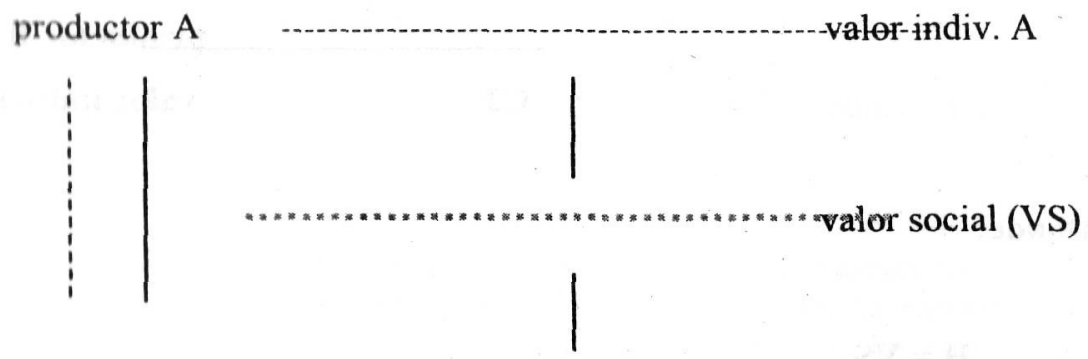

productor $\mathrm{B}$

valor indiv. B

\section{Como se forma VS?}

L1 proceso de demostración se basa en la aplicación del Método del Paralelogramo de Puerzas ${ }^{12}$

I I ste es un Método de la Física clásica que dice que cuando dos fuerzas interactúan entre sí, se obtiene, por el juego de acciones y reacciones entre las fuerzas interactuantes, una tercera que no es de suyo igual a las dos que le dieron origen, sino diferente. El método de medición no puede ser la simple suma de las fuerzas individuales. Para ello se aplica el conocido teorema de Pitágoras que dice que el cuadrado de la hipotenusa es igual a la suma de los euadrados de los catetos. El método aquí se utiliza sólo como un recurso analógico. Engels también lo fiza cuando intentó aclarar el significado de lo económico como determinante en última instancia (ver la carta de lingels a Bloch de 1890 y el trabajo de Althusser "Contradicción y Superdeterminación." Para nosotros las fieizas individuales están representadas por dos productores diferentes (o sea, en nuestro modelo explicativo la realidad ha sido reducida o simplificada a esas dos fuerzas) que al enfrentarse entre sí en el mercado generan esa 


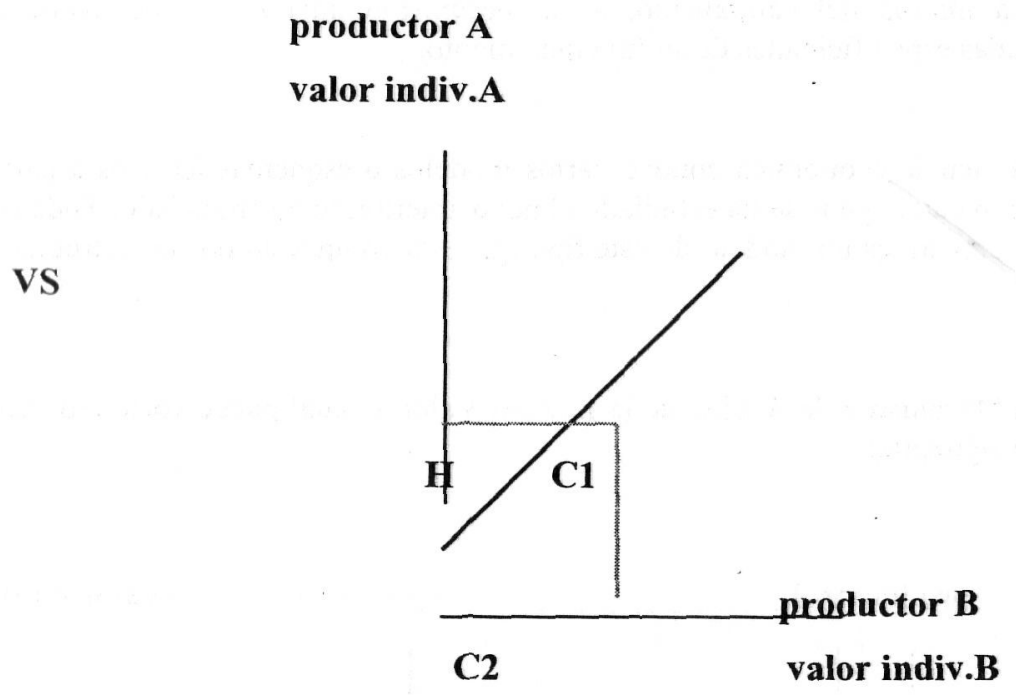

nde:

$$
\begin{aligned}
& H=V S \\
& C 1=V i a \\
& C 2=V i b
\end{aligned}
$$

icando Teorema de Pitágoras:

$$
\begin{array}{lc}
2 & 2 \\
= & C 1+C 2
\end{array}
$$

a fuerza (la fuerza del mercado) que no es la simple suma sino la resultante pitagórica interpretada aquí valor Social. 
$I I=\sqrt{\mathrm{C} 1+\mathrm{C} 2} 2$

$2 \quad 2$

$V S=(V i a)+(V i b)$

$V_{S}=\sqrt{(V i a)+(V i b)} 2$

El modelo, como representación simplificada de la realidad económica que intenta explicar, sugiere una serie de presupuestos:

P1: La realidad económica se reduce al momento de la producción (oferta), no incluye el momento del consumo (demanda). Supone, por tanto, la existencia de productores (A y B) privados, aislados e independientes los unos de los otros, todos tratando de lograr maximizar su función de producción.

P2: Las condiciones de producción son diferentes para los productores, aunque ambos producen para el mismo mercado. Este concepto (condiciones de producción) se desprende, evidentemente, de las características del modelo marxista siguiente:

$\operatorname{Mp}(k)$

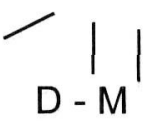

...P(y) $\ldots$

Ft (L) 
que como puede apreciarse se deriva, a su vez, del concepto Fuerzas Productivas, las que, como ya dijimos en parte anterior de este trabajo, se asocia al concepto de relaciones tecnológicas, las que en este caso concreto se articulan o combinan en una determinada proporción a los Mp (k) con la $\mathrm{Ft}(\mathrm{L})$, siendo esta combinación de los factores de la producción la que origina el concepto de Composición Orgánica del Capital (C.O.C) y dentro de ella, específicamente el concepto de Composición Técnica (C.T.), o sea, P es una Función de $\mathrm{Mp}$ y de $\mathrm{Ft}: \mathrm{P}=f(\mathrm{Mp}, \mathrm{Ft})$, lo que equivale a la clásica función de tipo Cobb - Douglas, donde $\mathrm{y}=f(\mathrm{k}, \mathrm{l})$. En este caso $\mathrm{CT}=\mathrm{Mp} / \mathrm{Ft}$ ó $\mathrm{k} / \mathrm{l}$.

En el gráfico que representa al mecanismo de acción de la ley del valor se puede apreciar claramente que VS $=f(\mathrm{Mp}, \mathrm{Ft})$ ó VS $=f(\mathrm{k}, 1)$, la que es diferente en A y en B, $[\mathrm{Via}=f(\mathrm{Mp}, \mathrm{Ft})>\mathrm{Vib}=f(\mathrm{k}, \mathrm{l})]$, y esto sólo es posible si se verifica la situación que a continuación muestra el gráfico siguiente:

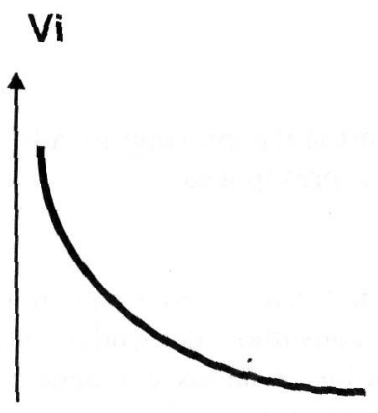

$(\mathbf{M} p / F t)$ ó $(\mathrm{k} / \mathrm{L})=\mathrm{C} . \mathrm{T}$. 
La curva muestra las variaciones que sufre $V i$ como consecuencia del erecimiento o decrecimiento de C.T.

\section{$\mathbf{V i}$}

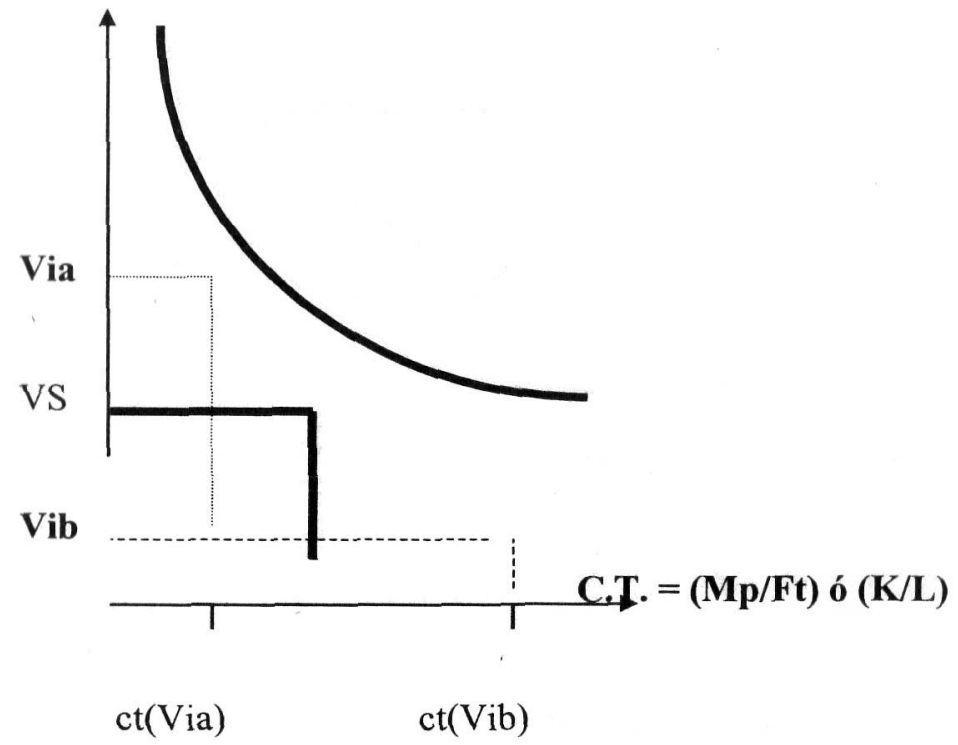




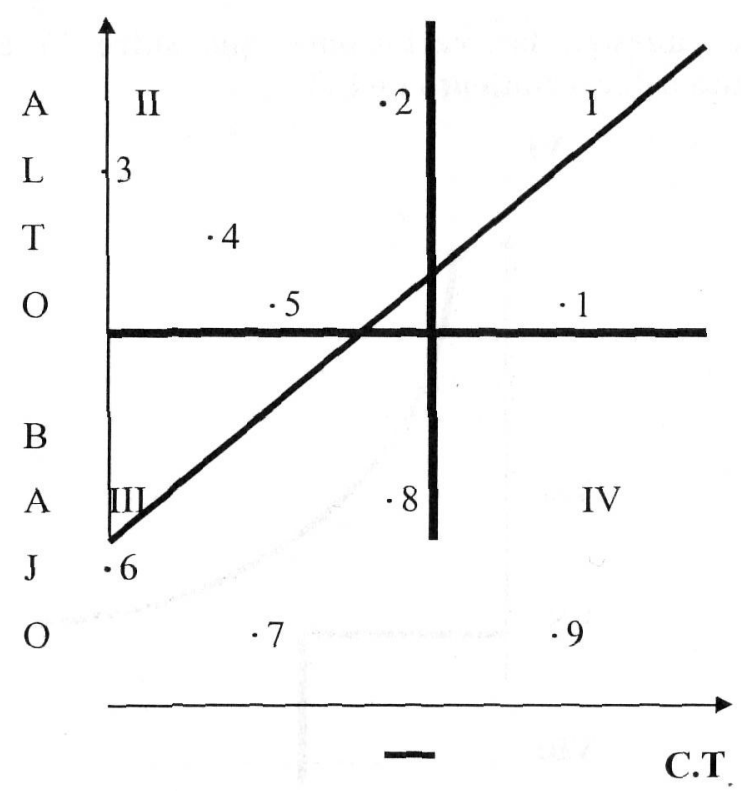

B A J A C.T. A L T A

uadrante I: Están los productores que tienen una alta C.T., pero al mismo tiempo tienen un alto Vi. Evidentemente que esta no es una buena posición, pues no se corresponde con lo expresado en el gráfico anterior. Todo lo contrario, si allí la curva tenía un sentido descendente, aquí lo tiene de modo ascendente, o sea, en la medida que crece la estructura técnica de la producción crece también el valor individual del producto, lo que es irracional desde el punto de vista económico.

uadrante II: Están los productores que tienen una baja C.T. y por ende, según lo mostrado en el gráfico anterior, un alto Vi, ya que éste es $f($ C.T.). De todas formas es bueno tener en cuenta que, incluso, dentro de este cuadrante la situación de los productores no es exactamente la misma. La distancia de cada uno de ellos de la diagonal refleja claramente la magnitud del problema. Mientras más grande sea la distancia absoluta entre un punto dentro del cuadrante y la línea diagonal, peor será la situación para el productor individual. Así por ejemplo, el productor 2 es el que tiene la peor situación de todos. Este será, posiblemente, el más vulnerable a la lucha competitiva con el resto de los productores $y$, por 
ende, el primero en arruinarse.

Curhtrante III: Están los productores que tienen baja C.T. y bajo Vi, lo que de acuerdo con gráfico anterior es normal, dado que $\mathrm{Vi}=f($ C.T.).

Cuadrante IV: Están los productores con alta C.T. y Vi bajo. Es esta situación la que se corresponde con el modelo desarrollado en el gráfico.

Si comparamos ambos gráficos podremos comprobar que Via, se ubica en los chiadrantes I y II, es decir, superiores al VS. Todo lo contrario ocurre con Vib, ubicado en los cuadrantes III y IV, es decir por debajo del VS. Ahora Bien, ¿Qué consecuencias se derivan de esta situación, que no representa otra cosa que el impacto que tiene el diferente desarrollo de las relaciones tecnológicas $(\mathrm{Fp})$ en las relaciones entre los productores ( $\mathrm{Rp}$ o estructura económica)?. Se pueden apreciar con relativa precisión, al menos, dos tipos diferentes de impactos (tendencias), pero estrechamente relacionados entre sí, a saber:

1. It diferente desarrollo tecnológico de los productores condiciona la existencia de $\mathrm{Vi}$ también diferentes. El mecanismo de acción de la ley del valor genera una tendencia a profundizar esta diferencia y con ella el proceso de diferenciación de los productores. ( omo resultado se produce de modo paulatino una cierta polarización que se expresa en el cnriquecimiento de unos (de aquellos que con mejores condiciones tecnológicas son capaces de producir por debajo del valor social: cuadrante IV) y la ruina y el empobrecimiento de otros (de aquellos que con peores condiciones tecnológicas, producen por encima del valor social: cuadrante II).

2. El diferente desarrollo tecnológico condiciona la necesidad de que los productores que lienen peores condiciones y que producen, por tanto valores individuales por encima del valor social desarrollen procesos de invención, innovación e introducción de los adelantos científico - técnicos para no perecer en la competencia con los demás. Esto último constituye un verdadero proceso de transferencia de tecnología, del polo más desarrollado hacia el menos desarrollado y que incluye todos los momentos que el concepto encierra:

a) El Paradigma y la Trayectoria Tecnológica

b) La Tecnología Disponible

c) La Apropiación (asimilación) de la Tecnología Disponible

d) La Capacidad Tecnológica

De modo tal que ello estimularía un movimiento en el sentido y dirección que aparece representado en el gráfico siguiente: 


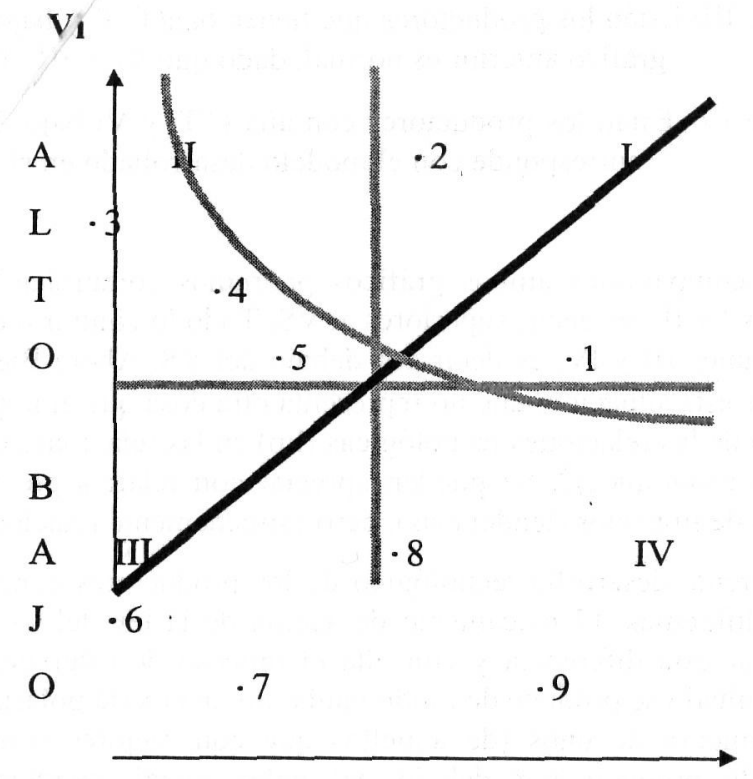

\section{BAJA C.T ALTA}

De Composiciones Tecnológicas bajas hacia Composiciones Tecnológicas altas, es decir del Cuadrante II hacia el Cuadrante IV. Es evidente que este proceso de difusión tecnológica genera el desarrollo de las Fuerzas Productivas (Relaciones Tecnológicas).

En estrecha relación con estas dos tendencias, se sitúan dos nociones muy importantes en cuanto al papel de la Ciencia y la Tecnología y de sus impactos en las relaciones sociales.

Desde la perspectiva de los productores que están en el cuadrante II el desarrollo tecnológico ocurre como resultado que su demanda ejerce una función dominante en la conducción de las invenciones, las innovaciones y la transferencia de tecnología. Este proceso se conoce como Demand - pull, es decir, es la demanda la que "hala" a la tecnología.

Desde la perspectiva de los productores que están en el cuadrante IV la cosa es 
bien difcrente, pues aquí son la ciencia y la tecnología las que impulsan a los productores hactia la busqueda de las invenciones, las innovaciones y la transferencia. Esto se conoce como Technology-push, es decir, la ciencia y la tecnología "empujan". El Technologypma $/ 4$ admite incluso otra interpretación consistente en considerar que la ciencia y la tecnologia se desarrollan de forma independiente al sistema económico. En este caso se considera a la ciencia exógena y neutra en interacción con la tecnología y la economía al ententer que el proceso de desarrollo científico desemboca de forma inexorable en una cierta tecnología.

\section{It) Modelo de Análisis del Ciclo del Capital Industrial}
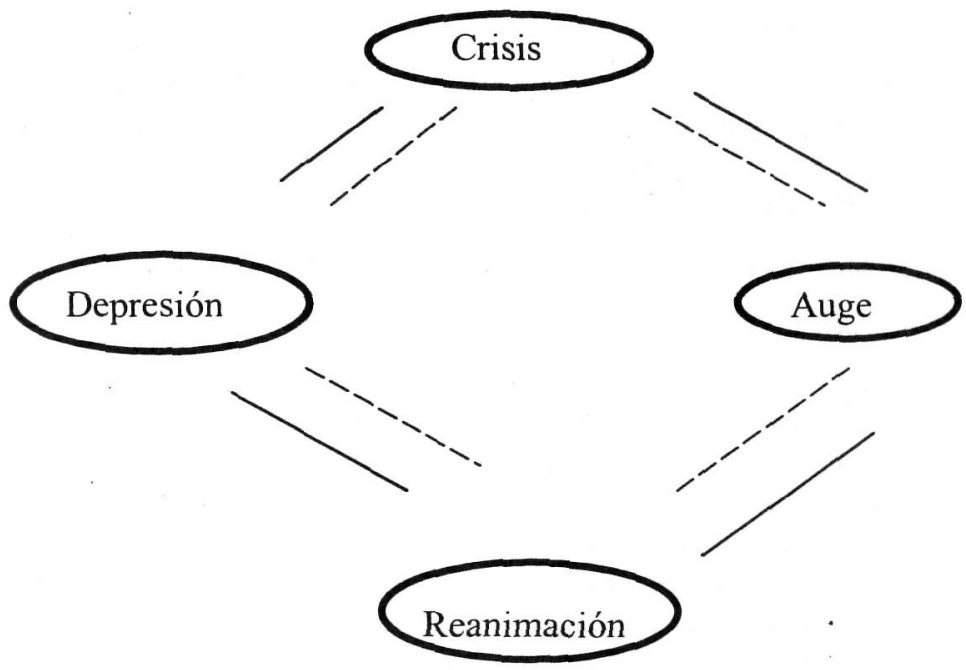

Donde se demuestra que la Renovación ${ }^{13}$ del Capital Fijo es la única vía para salir de la crisis. El mecanismo mediante el cual esto transcurre, fue explicado en el inciso anterior.

\footnotetext{
1. La Renovación (del Capital Fijo) exige desarrollar los procesos de Invención o innovación, apropiación tecnológica o transferencia de tecnología, teniendo en cuenta el Paradigma y las Trayectorias Tecnológicas existentes, la tecnología disponible y su apropiación, así como la capacidad tecnológica.
} 
El esquema o modelo utilizado permite explicar la problemática del desarrollo capitalista a partir del análisis de la interrelación que existe entre las relaciones económicas y las relaciones tecnológicas.

El Modelo de ra Tendencia Histórica de la Acumulación Capitalista, donde realmente se resume la idea expuesta en el Prólogo de la Contribución a la Crítica de la Economía Política en el sentido del nexo entre Relaciones de Producción (Económicas) y Fuerzas Productivas (Tecnológicas). Aquí se pueden apreciar, de manera más nítida, el mecanismo de polarización y la desigualdad del desarrollo capitalista, base para entender algunos procesos muy relacionados entre sí tales como:

a) La Concentración y Centralización del desarrollo científico y tecnológico en Centros de dominación, así como de las fuentes para su financiamiento.

b) El desarrollo económico y tecnológico desigual entre regiones y países lo cual sirve de base para el establecimiento de relaciones de dependencia y subordinación de los polos menos desarrollados a los más desarrollados.

Estos procesos se dan primeramente en el plano interno para luego, por la lógica inexorable del movimiento del capital desbordar las fronteras nacionales para reproducir a escala internacional el esquema anteriormente descrito. Todo ello es básico para poder. explicar el problema de la relación entre:

a) Desarrollo científico y tecnológico y subdesarrollo en el ámbito internacional

b) Desarrollo científico y tecnológico y subdesarrollo regional.

Con lógicas diferentes, pero con muchos puntos de contacto con el esquema de Marx, existen otros Modelos donde a partir de la relación desarrollo tecnológico desarrollo económico, se explica el funcionamiento del capitalismo actual y sus tendencias principales. Nos referimos, por ejemplo, al llamado Modelo de las Ondas del Capitalismo, que al igual que en el caso de las Teorías de los Ciclos demuestran las profundas transformaciones que sufrió el sistema económico capitalista gracias a las innovaciones que se implementaron a partir de los procesos competitivos ${ }^{14}$ para reducir los costos e incrementar las ganancias (economías de escala).

La Teoría de las Ondas del Capitalismo se basa en una conceptualización que refleja los impactos del desarrollo científico y tecnológico en las relaciones económicas y demás esferas de la vida social en el capitalismo y ha tenido, por eso, una amplia divulgación en la literatura

especializada asumiendo las más diversas formas de expresión destacándose las así llamadas Ondas Cortas de Kitchin, las Ondas Largas de Kondrátiev (ambas surgidas aproximadamente en 1928), de Schumpeter, Mandel y Freeman, que estiman estas ondas en ciclos largos de 50 años, así como la Teoría sobre Ondas más Largas o Eras de Kaplinski.

\footnotetext{
${ }^{14}$ Estos procesos se dan entre fracciones del capital y entre el capital y el trabajo.
} 
La utilización de estas teorías en la explicación del desarrollo capitalista ha servido de base a diferentes conclusiones:

1. La conclusión marxista que demuestra, en el Modelo Teórico, el desarrollo lesenologico como un resultado del desarrollo capitalista a la vez que condicionante del cambie o sustitución de éste por un sistema económico más avanzado.

2. I a conclusión shumpeteriana, que demuestra la capacidad de adaptabilidad del capitatismo utilizando, precisamente las bondades del desarrollo tecnológico.

3. La conclusión rostowiana, que recogiendo la tradición liberal, hegeliana, Lojeviana y finalmente, fukuyamiana, formula la Teoría de las Fases del Crecimiento f conomico, alternativa al Modelo marxista, donde se emite un mensaje de esperanza al polo menos desarrollado al crear la expectativa de que su situación actual forma parte del desarrollo natural por el que algunos pasaron y por donde los demás deberán pasar. Posición reforzada hoy por el derrumbe, desmantelamiento y transición hacia el capitalısmo del llamado socialismo real este europeo, así como por las reformas de mercado que otros países como Cuba, China, Vietnam, etc. adoptan para salir de sus respectivas crisis económicas.

Ahora bien, lo común a todos los Modelos y Teorías (incluyendo el marxismo), es que están asociados a grandes cambios en la Ciencia y la Tecnología, comúnmente conceptualizados como Revoluciones.

Estas Revoluciones han afectado a los cuatro pilares fundamentales del desarrollo tecnológico: la energía, los materiales, los seres vivos y la información.

En cuanto a la energía. Lo primero que habría que señalar es que toda onda, ciclo, cra o Revolución siempre tiene como base una determinada fuente de energía. Según algunos autores, la Primera Revolución Tecnológica (RT) de la era Moderna, se inició entre 1787 y 1827 y se basó en el carbón como fuente principal, la que también es común a la Segunda Revolución Tecnológica que se sitúa de 1828 a 1885.

Ambas RT se corresponden con el estadío o fase desarrollo del capitalismo de libre competencia en dos niveles diferentes. Un primer nivel de desarrollo hacia dentro de Europa y un segundo nivel hacia afuera, en busca de mercados y países donde colocar los excedentes, primero mercantiles y luego de capital, y de donde obtener la materia prima indispensable que dicho desarrollo demandaba. Así se fueron articulando al proceso toda una serie de paises en condición de colonias, ocupando el lugar que la División Internacional del Trabajo formada bajo esas exigencias les asignó como destino histórico.

Aún no se puede hablar aquí de contradicción desarrollo subdesarrollo, pero ya se están sentando las bases. Marx califica a esta relación como adelanto (avance) y atraso, dando la idea de que este desbordamiento del capitalismo más allá de sus fronteras generaba un desarrollo tecnológico en los países receptores y que por tanto era posible que estos ganaran la brecha que los separa de sus metrópolis desarrolladas. Esto es lógico, pues aún el desarrollo económico y político desigual del capitalismo no se ha manifestado 
mo ley objetiva, sino sólo como diferencia.

La tercera RT (1886 - 1936) y la cuarta (1939 - 1995), han tenido como fuente de rrgía el petróleo y se ubican en la fase de desarrollo imperialista del capitalismo. Es en a fase donde la relación entre avance y atraso se transforma en la relación desarrollo idesarrollo. En el gráfico que a continuación se muestra refleja lo dicho anteriormente:

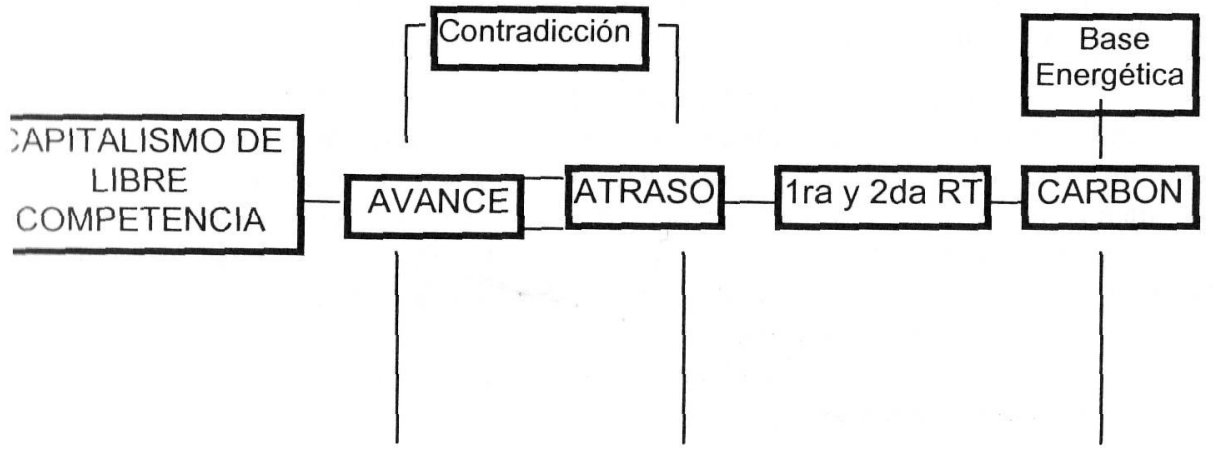

Se habla también de una quinta RT, que al igual que las demás se gesta en el seno । anterior. Su base energética fundamental sería la energía atómica, tendiendo más, y rimera vez, hacia la microelectrónica y sus aplicaciones así como a la información, jon las que determinan la dinámica de todo el sistema económico mundial. De ahí que sculiaridad, según el criterio de algunos autores, esté en que las innovaciones que la :terizan no tienen un sólo eje.

En cuanto a los materiales el principal cambio que se aprecia se refiere a lo que se ado en llamar la desmaterialización del proceso productivo. Este es un fenómeno o que tiene serias implicaciones en el orden de las relaciones económicas y humanas 
en sencral. El proceso de la desmaterialización se pone de manifiesto a través de los imincmtos siguientes:

1. El creciente papel de la esfera de los servicios de pre-venta, producción, venta y post-venta. Estos servicios, aunque están soportados materialmente, de por sí se perciben como actividad, desempeño, relación directa entre proveedor y cliente que es lo que se produce y consume.

2. El proceso de reducción sistemática de las materias primas y materiales por unndad de producto o servicio, sustentado no sólo en el diseño de sistemas de producción cadia vez más eficientes, sino en un proceso paulatino de sustitución de los productos labricados con materiales de origen natural por materiales sintéticos. El descubrimiento de nucvos insumos y materiales, que sustituyen a los tradicionales, está conformando un uıc vo paradigma de desarrollo económico con implicaciones muy negativas para el resto de los paises que objetivamente no pueden articularse al tren del desarrollo tecnológico, a pesar de la esperanza rostowiana.

3. El creciente aumento de mercados de productos asociados a la industria de la unformática. Hoy se conoce y se acepta, que aunque los sistemas de software están soportados en un sistema eminentemente material, ellos son tan intangibles y dependen lanto de la inteligencia o de la labor intelectual de los hombres que difícilmente ellos unismos sean calificados como productos materiales. Este problema está impactando no sólo en las formas de comunicación social, sino también en los propios sistemas de valores subjetivos e instituidos de las sociedades contemporáneas. Nuevos conceptos cconómicos y jurídicos han invadido las constituciones de los países, donde quizás el más connotado sea el de la así llamada propiedad intelectual y su protección.

Estos procesos se vienen verificando fundamentalmente entre la cuarta y la quinta RT, las que se corresponden sincrónicamente con el fortalecimiento de la relación entre desarrollo y subdesarrollo. Por eso ahora señalaremos algunos de los impactos que sobre la misma tienen los cambios tecnológicos apuntados.

Las estructuras económicas en los países subdesarrollados se han formado históricamente de modo tal, que no se conciben sino estrechamente articuladas a Modelos de desarrollo sustentados en fuentes de energía no renovables y en la producción de productos primarios para la exportación. En este caso la función capital/trabajo $[f(\mathrm{~K}, \mathrm{~T})]$ se corresponde con la situación representada en la Zona II del gráfico mostrado en la página 12 es decir, una zona de baja CT.

Como consecuencia lógica este Modelo presupone la existencia de una fuerza de trabajo, que como regla no requiere de altos niveles de calificación. Es también un Modelo estrechamente articulado a las exigencias de los procesos productivos de los centros, subordinado a él y formando parte de él. Sin embargo nótese que producto de los cambios apuntados el sincronismo tradicional del ciclo se va quebrando por el hecho de que aquellos vienen acompañados de una nueva división del trabajo basada en nuevas fuentes de energías y en la producción de materiales y materias primas sintéticos, que 
desplazan paulatinamente a los proveedores tradicionales, los que por primera vez ven cómo sus exportaciones tradicionales se deprecian y ellos mismos son cada vez menos necesarios al nuevo paradigma que se viene conformando: ni sus productos ni sus gentes.

La fuerza de trabajo, diseñada para un Modelo económico en decadencia, va siendo cada vez menos importante, perdiendo así su capacidad negociadora frente a la fuerza demoledora del gran capital. El principal impacto, desde la perspectiva de lucha de clase obrera, tanto del centro como de la periferia, sería la reevaluación del tradicional concepto de explotación.

La asincronía se profundiza también por el hecho de que los centros desarrollados han transitado ya de los sistemas de producción en masa y de las economías de escala, de inspiración fordista-taylorista a sistemas flexibles e integrados de producción orientados hacia el consumidor, donde la segmentación y la supersegmentación (ilustres) así como los modelos cero defectos (Just in time, Kan ban, MRP, por ejemplo) son sus características fundamentales.

La periferia subdesarrollada aún no logra siquiera rebasar la modernidad económica tradicional, donde el fordismo, el taylorismo, la producción en masa y las economías de escala conforman los ingredientes fundamentales de ese paradigma tecnológico moderno.

Mostramos cómo el desarrollo tecnológico es un resultado del desarrollo capitalista, a la vez que condición de la sustitución de éste por un sistema más avanzado, en contraposición a la conclusión shumpeteriana, que afirma la capacidad de adaptabilidad del sistema utilizando, precisamente, las bondades del desarrollo tecnológico.

El modelo de Marx es un instrumento de carácter metodológico para poder entender las desigualdades regionales en cuanto a pobreza y distribución de renta. 
1.IMOS, A. Investigación sobre Desarrolo Humano en el Extremo Norte de Brasil. lesis de Doctorado. Universidad de Matanzas, 1999.

I.ININ, V. I. Quiénes son los Amigos del Pueblo y cómo Luchan Contra la Socialdemocracia. Moscú: Editorial Progreso, 1978.

MARX, K. Capital: A Critique of Political Economy, Vol. 3, F. Engels, ed., Moscow: lioreign Languages Publishing, 1962.

MARX, K. Para la Crítica de la Economia Politica. Moscú: Editorial Progreso, 1973.

MARX, K. Ideologia Alemana. La Habana: Editora Politica, 1979.

RIMA, I. H. Development of Economic Analysis. London: Routledge, 1996.

SCHUMPETER, J. A. Teoria do Desenvolvimento Econômico: Uma Investigação sobre Lucros, Capital, Crédito, Juro e o Ciclo Econômico. São Paulo: Abril Cultural, 1982.

SIMONSEN, M. H. Ensaios Analíticos. Rio de Janeiro: Editora da Fundação Getulio Vargas, 1994.

SMITH, A. An Inquiry into the Nature and Causes of the Wealth of Nations. New York: Modern Library, 1937.

SOUZA, N. J. Desenvolvimento Econômico. São Paulo: Atlas, 1997. 